\title{
Clinical characteristics of portal hypertension compli- cated by gastroesophageal varices in patients with myeloproliferative neoplasms
}

\author{
Jaejun Lee ${ }^{1,2}$, Pil Soo Sung ${ }^{1,2}$, Ki-Seong Eom ${ }^{3}$, Hyun Yang ${ }^{1,2}$, Soon Kyu Lee ${ }^{1,4}$, Aung Hlaing Bwa ${ }^{5}$, Angelo Lozada ${ }^{6}$, \\ Jeong Won Jang ${ }^{1,4}$, Si Hyun Bae ${ }^{1,2}$, Jong Young Choi ${ }^{1,4}$, and Seung Kew Yoon ${ }^{1,4}$ \\ ${ }^{1}$ The Catholic University Liver Research Center, College of Medicine, The Catholic University of Korea, Seoul; ${ }^{2}$ Division of Hepatology, \\ Department of Internal Medicine, Eunpyeong St. Mary Hospital, College of Medicine, The Catholic University of Korea, Seoul; Divisions \\ of ${ }^{3}$ Hematology and ${ }^{4}$ Hepatology, Department of Internal Medicine, Seoul St. Mary's Hospital, College of Medicine, The Catholic Univer- \\ sity of Korea, Seoul, Korea; ${ }^{5}$ Department of Hepatology, Yangon GI \& Liver Center, Yangon, Myanmar; ${ }^{6}$ Department of Internal Medi- \\ cine, Makati Medical Center, Manila, Philippines
}

Dear Editor,

Philadelphia chromosome-negative myeloproliferative neoplasms (MPNs) which include polycythemia vera (PV), essential thrombocythemia (ET), and primary myelofibrosis (PMF) are characterized by erythrocytosis, leukocytosis, thrombocytosis, bone marrow hypercellularity, splenomegaly, and extramedullary hematopoiesis. ${ }^{1}$ A rare complication of Philadelphia-negative MPNs is portal hypertension (PHT). Previous reports have indicated that the incidence of PHT in patients with MPN is approximately $7 \%$ to $18 \%{ }^{2}$ The first manifestation of the disease may be complications of PHT, such as bleeding gastroesophageal varices (GEVs). ${ }^{3}$ Typically, endoscopic variceal ligation and sclerotherapy is used to control GEVs, and a transjugular intrahepatic portosystemic shunt (TIPS) is applied for variceal bleeding or refractory ascites. ${ }^{4}$

MPNs are often associated with Janus kinase 2 (JAK2) V617F mutations. ${ }^{5}$ JAK2 is a nonreceptor tyrosine kinase expressed by all hematopoietic stem cells; it relays the signal to induce cell proliferation when cells are stimulated by external cytokines or growth factors. ${ }^{5,6}$ The JAK2 V617F mutation leads to the constitutive phosphorylation of JAK2 and stimulates the cell proliferation cascade and the clonal proliferation of hematopoietic cells. ${ }^{6}$ The JAK2 V617 mutation may be a risk factor for splanchnic circulation thrombosis in patients with MPN and subclinical MPN, although the detailed mechanism has not been elucidated. ${ }^{2}$ An increased

\section{Abbreviations:}

CT, computed tomography; ET, essential thrombocythemia; GEV, gastroesophageal varices; HVPG, hepatic venous pressure gradient; JAK2, Janus kinase 2; LDLT, living donor liver transplantation; MPN-U, myeloproliferative neoplasm unclassified; MPN, myeloproliferative neoplasm; PHT, portal hypertension; PMF, primary myelofibrosis; PV, polycythemia vera; TIPS, transjugular intrahepatic portosystemic shunt

\section{Corresponding author: Pil Soo Sung}

Division of Hepatology, Department of Internal Medicine, Eunpyeong St. Mary Hospital, College of Medicine, The Catholic University of Korea, 1021 Tongil-ro, Eunpyeong-gu, Seoul 03312, Korea

Tel: +82-2-2030-2530, Fax: +82-2-3481-4025

E-mail: pssung@catholic.ac.kr

https://orcid.org/0000-0002-5780-9607

\section{Ki-Seong Eom}

Division of Hematology, Department of Internal Medicine, Seoul St. Mary Hospital, College of Medicine, The Catholic University of Korea, 222 Banpo-daero, Seocho-gu, Seoul 06591, Korea

Tel: +82-2-2258-2073, Fax: +82-2-3481-4025

E-mail: dreom@catholic.ac.kr

https://orcid.org/0000-0002-0578-126X 
JAK2 mutation frequency in splanchnic circulation thrombosis or extramedullary hematopoiesis is associated with a high PHT incidence in patients with MPN. However, it is not clear whether specific subgroups of patients with MPN are more susceptible to the development of PHT or whether the JAK2 V617F mutation is associated with PHT in patients with MPN.

In this retrospective study with a large cohort of MPNs, we evaluated the clinical characteristics of patients with PHT complicated by GEVs and Philadelphia-negative MPNs. Most previous reports of PHT in MPN are case reports or case series, and detailed clinical characteristics of patients are generally lacking. ${ }^{1,6-8}$ The mechanisms underlying PHT in MPNs are still unclear. Increased blood flow into the portal system through the enlarged spleen is one of the primary causes of the development of PHT. The intrahepatic obstruction of the portal system due to myeloid metaplasia or sinusoidal change also increases portal pressure. ${ }^{9}$ Thrombosis of the portal vein due to blood vessel endothelium damage also induce $\mathrm{PHT} .^{10}$

We designed a retrospective single-center study. This study was approved by the Institutional Review Board of Seoul St. Mary's Hospital (KC19RESI0476). The medical records of all patients diagnosed with MPNs at Seoul St. Mary's Hospital between January 2009 and December 2018 were reviewed. BCR-ABL1-negative MPNs primarily include PV, ET, PMF, and myeloproliferative neoplasm unclassified (MPN-U). The clinical diagnosis of PV, ET, PMF, and MPN-U was conducted in accordance with the 2016 WHO classifications."

Normal hepatic venous pressure gradient (HVPG) is typically 1 to $5 \mathrm{mmHg}$, and significant PHT is considered as an increase in $H V P G \geq 10 \mathrm{mmHg}$, leading to the development of complications of $\mathrm{PHT}^{12}$ Owing to the inability to measure HVPG or to perform endoscopy, PHT was evaluated by the existence of GEVs, as verified by abdominal computed tomography (CT). An esophageal varix was radiologically defined as an enhancing nodular tubular structure protruding into the esophageal lumen. A previous report demonstrated that $\mathrm{CT}$ showed a $90 \%$ sensitivity in the detection of large ( $>5 \mathrm{~mm}$ in diameter) GEVs. ${ }^{13}$ In our study, to increase the specificity of clinically significant GEV detection, the threshold diameter for esophageal varix was set as $5 \mathrm{~mm}$ on abdominal CT. Data were collected at the time of the abdominal CT. Quantitative variables are expressed as medians (interquartile range), whereas percentages are reported for qualitative data. Comparisons between groups were performed using the Mann-Whitney test. Values of $P<0.05$ were considered statistically significant.

Two hundred and twenty eight patients with MPN underwent contrast-enhanced abdominal CT at least once between January 2009 and December 2018 (Table 1, 2). Among these 228 patients, 50 (21.9\%) were diagnosed with PV, 72 patients (31.6\%) had ET, 84 patients (36.8\%) had PMF, and 22 patients (9.7\%) did

Table 1. Baseline patient characteristics

\begin{tabular}{lcccc}
\hline & \multicolumn{4}{c}{ Total patients (n=228) } \\
\cline { 2 - 5 } & PV & ET & PMF & MPN-U \\
\hline Patients & $50(21.9)$ & $72(31.6)$ & $84(36.8)$ & $22(9.7)$ \\
PHT by GEVs & $5(10.0)$ & $2(2.8)$ & $3(3.6)$ & $1(4.5)$ \\
JAK2 V617F mutation & \multicolumn{4}{c}{$130(57.0)$} \\
Positive & $34(68.0)$ & $37(51.4)$ & $47(56.0)$ & $10(45.5)$ \\
Negative & 16 & 34 & 37 & 12 \\
Not available & 0 & 1 & 0 & 0 \\
\hline
\end{tabular}

Values are presented as number (\%).

$P V$, polycythemia vera; ET, essential thrombocythemia; PMF, primary myelofibrosis; MPN-U, myeloproliferative neoplasms-unclassified; PHT, portal hypertension; GEV, gastroesophageal varices.

Table 2. Clinical characteristics of patients with PHT

\begin{tabular}{lc}
\hline & Value \\
\hline $\begin{array}{l}\text { Patients with PHT by GEVs } \\
\text { (n=11, } 5 \% \text { of total patients) }\end{array}$ & \\
PV & $5(45.5)$ \\
ET & $2(18.2)$ \\
PMF & $3(27.3)$ \\
MPN-U & $1(9.1)$ \\
Median age & 60 \\
Male & $5(45.5)$ \\
Anti-HCV positivity & $0(0)$ \\
HBsAg positivity & $0(0)$ \\
Portal vein thrombosis & $3(27.3)$ \\
JAK2 V617F mutation & $11(100)$ \\
Median total bilirubin (mg/dL) & $1.54(0.68-2.53)$ \\
Median albumin (g/dL) & $4.3(3.6-4.8)$ \\
\hline Median INR & $1.25(1.15-1.60)$ \\
\hline Treatment & \\
Ruxolitinib & 2 \\
Hydroxyurea & 7 \\
\hline
\end{tabular}

Values are presented as median (range) or number (\%).

PHT, portal hypertension; GEV, gastroesophageal varices; PV, polycythemia vera; ET, essential thrombocythemia; PMF, primary myelofibrosis; MPN-U, myeloproliferative neoplasms-unclassified; $\mathrm{HCV}$, hepatitis C virus; $\mathrm{HBsAg}$, hepatitis $B$ surface antigen; JAK2, Janus kinase 2 ; INR, international normalized ratio. 
not meet the criteria for these three diseases and were classified as MPN-U. A total of 11 patients among 228 patients had GEVs, as confirmed by abdominal CT. A total of 130 out of 228 patients had the JAK2 V617F mutation and all patients with GEV had the JAK2 V617F mutation ( $57 \%$ vs. $100 \%, P=0.003$ ). The incidence of the JAK2 V617F mutation in each group was as follows: $68 \%$ (34/50), PV; 52\% (37/72), ET; 56\% (47/84) PMF; 45\% (10/22), MPN-U.

Among 11 patients having GEVs, five had PV, two had ET, three had PMF, and the remainder were classified as having MPN-U. The median age was 60, and five subjects were male. The median spleen size was $16.9 \mathrm{~cm}$. No patients had chronic viral hepatitis or other chronic liver diseases such as hemochromatosis or autoimmune hepatitis (Table 1, 2). Three had portal vein thrombosis without abnormalities in coagulation factor assays. All these patients were evaluated for the liver function (international normalized ratio, albumin, total bilirubin, presence of ascites, and presence of hepatic encephalopathy), and were categorized into ChildPugh class A, indicating that the liver function was well-preserved (Table 1, 2). Three patients had variceal bleeding, which had not been lethal and controlled by endoscopic ligation. Seven patients received hydroxyurea and two patients received ruxolitinib (a JAK1, 2 inhibitor), but both of the medication resulted in no changes in variceal size by CT imaging.

A 53-year-old patient was diagnosed with PMF in 2016 at Seoul St. Mary's Hospital. Ruxolitinib treatment was started after the diagnosis. On August 4, 2017, he visited the emergency room presenting with hematemesis, and esophageal varix bleeding was documented. The bleeding was successfully stopped by endoscopic ligation. Prominent varix formation (F3) was detected and TIPS was performed on August 10, 2017. In September of 2017, he developed hepatic encephalopathy and visited the hospital again. He and his family decided to undo the shunt and TIPS closure was performed on September 25, 2017. Within 1 month of the procedure, the patient presented with recurrent hematemesis. Esophageal varix bleeding was noted again and successfully ligated by endoscopy. The disease activity of PMF was stable, and the patient underwent successful living donor liver transplantation (LDLT) on November 24, 2017.

About 1 month after LDLT, in December of 2018, endoscopy revealed that varices were nearly resolved (Fig. 1A, B). We reviewed histologic findings of the recipient liver (Fig. 1C). We detected dif-
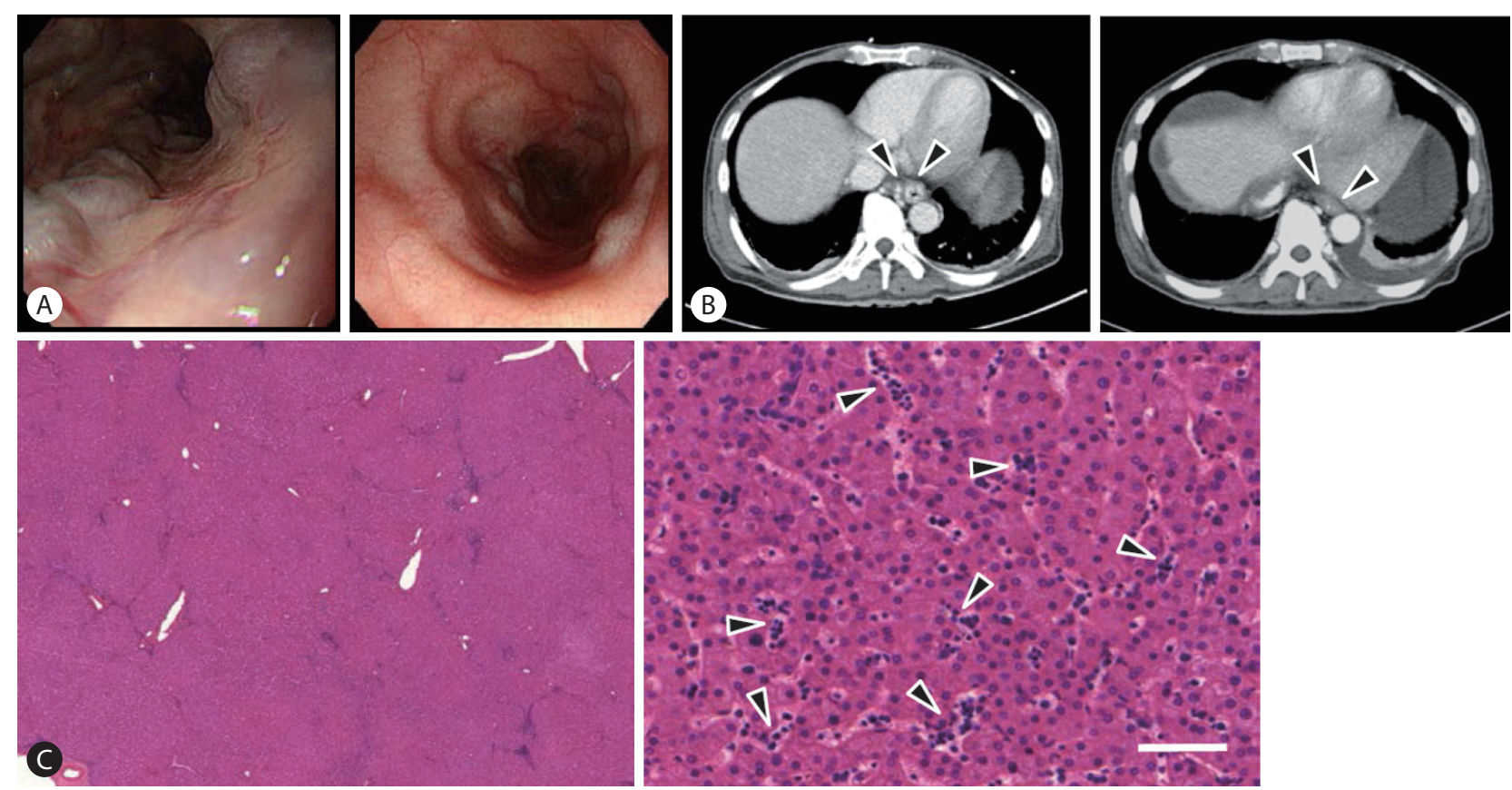

Figure 1. Regression of GEVs after liver transplantation in a patient with PMF. (A) Images of esophago-gastroduodenoscopy. Left panel represents the lumen of esophagus before LDLT and right panel represents after LDLT. (B) Contrast-enhanced CT scan of the abdomen. Left panel is an axial view of GEV before LDLT. Right panel is an axial view of GEV after LDLT. Arrowheads depict GEVs on CT scan. (C) H\&E stained histopathological images of explant liver. Left image is a low-power view of H\&E sections of the explant $(H \& E, \times 10)$. Right image is a high-power field image (H\&E, $\times 240)$. Scale bar 100 um (arrowheads: myeloid metaplasia at sinusoids). GEV, gastroesophageal varices; PMF, primary myelofibrosis; LDLT, living donor liver transplantation; CT, computed tomography; H\&E, Hematoxylin and eosin. 
fuse myeloid metaplasia at sinusoids, verifying the previous hypothesis based on pathophysiology that extramedullary myeloid metaplasia plays an important role in PHT of MPNs. By Masson's trichrome staining, we found a lack of fibrotic changes in the liver parenchyma, indicating that liver parenchymal injury is not the main pathophysiological factor.

Our analysis shows that about $5 \%$ of patients with MPN who underwent abdominal $\mathrm{CT}$ exhibited PHT, and three among 11 patients with PHT had variceal bleeding. Interestingly, our present study showed a strong correlation between the JAK2 V617F mutation and PHT. In our study, however, ruxolitinib did not have promising results for the treatment of variceal bleeding. One patient had recurrent variceal bleeding and underwent LDLT, which dramatically decreased portal pressure.

Several previous studies have demonstrated an association between the JAK2 V617F mutation and PHT and/or portal vein thrombosis. ${ }^{1,14}$ It is possible that the blood flow velocity in portal venous systems is slow, leading to prolonged interactions between blood and endothelial cells. Increased blood cells due to the clonal expansion by JAK2 V617F mutation could provoke thrombosis in the portal venous system. Hydroxyurea and JAK inhibitors are primary treatment options in MPNs. In a previous report on a patient who received ruxolitinib for PMF, PHT improved significantly, allowing discontinuation of all medications for ascites and GEVs. ${ }^{9}$ However, another report showed no effect of hydroxyurea and ruxolitinib on regression of $\mathrm{PHT}^{2}$ Our data also showed no effect of these drugs on the size change of GEVs. The discrepancy may stem from the degree of PHT. In our study, we defined PHT as having GEVs more than $5 \mathrm{~mm}$ on $\mathrm{CT}$, which may reflect severe PHT by MPNs.

Our study had some limitations, including the retrospective design and the use of abdominal $\mathrm{CT}$ to detect PHT, rather than HVPG measurement. However, owing to the inconvenience and invasiveness of the procedure, we could not recommend it for every patient.

In summary, patients with MPN can develop PHT and lifethreatening events such as variceal bleeding, particularly when the patient has JAK2 V617F mutation. In one patient, LDLT resolved PHT, suggesting that liver transplantation, rather than JAK inhibitors alone, is a therapeutic candidate when MPNs are wellcontrolled. Our findings also suggest that gastrointestinal endoscopy or abdominal CT should be performed to evaluate PHT in patients with Philadelphia-negative MPNs.

\section{Authors' contributions}

Jaejun Lee, Pil Soo Sung, and Ki-Seong Eom contributed to conception, design, acquisition, analysis, and interpretation of data. Aung Hlaing Bwa, Angelo Lozada, Hyun Yang and Soon Kyu Lee contributed to acquisition and interpretation of data. Jeong Won Jang, Si Hyun Bae, Jong Young Choi, and Seung Kew Yoon contributed to conception and design. Jaejun Lee and Pil Soo Sung wrote the manuscript.

\section{Financial support}

This study was supported by The Research Supporting Program of The Korean Association for the Study of the Liver and The Korean Liver Foundation (P.S.S). This research was also supported by the Basic Science Research Program through the National Research Foundation of Korea (NRF) funded by the Ministry of Education (NRF-2019R1I1A1A01059642).

\section{Conflicts of Interest}

The authors have no conflicts to disclose.

\section{REFERENCES}

1. Zhao G, Wu ZY, Zhang B, Sun YW, Luo M. Diagnosis and treatment of portal hypertension secondary to myeloproliferative disorders: a report of three cases. J Dig Dis 2011;12:312-316.

2. Yan M, Geyer H, Mesa R, Atallah E, Callum J, Bartoszko J, et al. Clinical features of patients with Philadelphia-negative myeloproliferative neoplasms complicated by portal hypertension. Clin Lymphoma Myeloma Leuk 2015;15:e1-e5.

3. Plaz Torres MC, Romana C, Coppo C, Zentilin P, Grillo F, Savarino V, et al. Education and imaging. Hepatobiliary and pancreatic: a rare cause of portal hypertension. J Gastroenterol Hepatol 2016;31:11.

4. Seo YS. Prevention and management of gastroesophageal varices. Clin Mol Hepatol 2018;24:20-42.

5. Foucar CE, Stein BL. JAK2 V617F mutation testing in patients presenting with hepatic and portal vein thrombosis. JAMA 2017;317: 2228-2229.

6. Klein S, Rick J, Lehmann J, Schierwagen R, Schierwagen IG, Verbeke $L$, et al. Janus-kinase-2 relates directly to portal hypertension and to complications in rodent and human cirrhosis. Gut 2017;66:145-155.

7. Abu-Hilal M, Tawaker J. Portal hypertension secondary to myelofibrosis with myeloid metaplasia: a study of 13 cases. World J Gastroenterol 2009;15:3128-3133.

8. Wiest R, Strauch U, Wagner $H$, Strotzer M, Woenckhaus M, Schröder $G$, et al. A patient with myelofibrosis complicated by refractory 
ascites and portal hypertension: to tips or not to tips? A case report with discussion of the mechanism of ascites formation. Scand J Gastroenterol 2009;39:389-394.

9. Tan HK, Leow WQ, Chang PE. Ruxolitinib for the treatment of portal hypertension in a patient with primary myelofibrosis. Gastroenterology 2019;157:e26-e27.

10. Leonardi F, Maria N, Villa E. Anticoagulation in cirrhosis: a new paradigm? Clin Mol Hepatol 2017;23:13-21.

11. Arber DA, Orazi A, Hasserjian R, Thiele J, Borowitz MJ, Le Beau MM, et al. The 2016 revision to the World Health Organization classification of myeloid neoplasms and acute leukemia. Blood 2016;127:2391-2405.
12. Bandali MF, Mirakhur A, Lee EW, Ferris MC, Sadler DJ, Gray RR, et al. Portal hypertension: imaging of portosystemic collateral pathways and associated image-guided therapy. World J Gastroenterol 2017;23:1735-1746.

13. Perri RE, Chiorean MV, Fidler JL, Fletcher JG, Talwalkar JA, Stadheim $\mathrm{L}$, et al. A prospective evaluation of computerized tomographic (CT) scanning as a screening modality for esophageal varices. Hepatology 2008;47:1587-1594.

14. How J, Zhou A, Oh ST. Splanchnic vein thrombosis in myeloproliferative neoplasms: pathophysiology and molecular mechanisms of disease. Ther Adv Hematol 2017;8:107-118. 